\title{
Quaderni
}

QUADERN I Communication, technologies, pouvoir

67 | Automne 2008

Jeu vidéo et discours

\section{Maîtrise du jeu, contrôle de soi : une quête à corps perdu}

Sylvie Craipeau, Gérard Dubey et Raphaël Koster

\section{OpenEdition}

\section{Journals}

Édition électronique

URL : http://journals.openedition.org/quaderni/228

DOI : 10.4000/quaderni.228

ISSN : 2105-2956

\section{Éditeur}

Les éditions de la Maison des sciences de l'Homme

\section{Édition imprimée}

Date de publication : 5 octobre 2008

Pagination : 85-92

\section{Référence électronique}

Sylvie Craipeau, Gérard Dubey et Raphaël Koster, « Maîtrise du jeu, contrôle de soi : une quête à corps perdu », Quaderni [En ligne], 67| Automne 2008, mis en ligne le 05 octobre 2008, consulté le 19 avril 2019. URL : http://journals.openedition.org/quaderni/228 ; DOI : 10.4000/quaderni.228 


\section{Communication}

\section{maîtrise du jeu, contrôle de soi :}

Les jeux vidéo, jeux sur console, sur PC, jeux d'arcade, jeux en ligne, se développent depuis les années soixante-dix. Apparus sur les campus américain et anglais à cette époque, ces jeux se sont considérablement développés, touchant un une quête à corps perdu public de plus en plus divers (les femmes sont aujourd'hui concernées avec les SIMS en particulier), mobilisant une technologie de plus en plus complexe. Ils ne relèvent plus d'une pratique marginale mais témoignent d'un phénomène culturel. La diffusion massive des jeux vidéo, surtout en ligne depuis le milieu des années quatre-vingt-

Sylvie Craipeau

Télécom Sud Paris

\section{Gérard Dubey}

Télécom Sud Paris

\section{Raphaël Koster}

\author{
Télécom Sud Paris \\ CETCO-PRA
} dix, s'accompagne d'un questionnement alarmé des familles et, en écho (ou est-ce le contraire ?) de la presse. Mais l'accent mis sur les phénomènes d'addiction aux jeux électroniques risque de masquer les véritables enjeux sociétaux attachés à ces pratiques nouvelles. C'est l'une des idées forces défendues dans cet article. Sous l'addiction il est au fond question de transformations du rapport à la règle, d'anomie, ce « mal de l'infini » identifié par Durkheim au siècle dernier, de transfert du contrôle social à des dispositifs techniques, de résistance enfin des corps et des principes de régulation sociale à ce processus de réification. Effet de la toute puissance de la règle plutôt que d'une dérégulation, produit de l'extension de la rationalité économique aux pratiques informelles, l'addiction aux jeux se révèle bien plus riche en significations que ce qu'une approche étiologique laisse accroire.

\section{Fabrique à bonheur versus fabrique à addictions}

Le qualificatif d'addiction peut se révéler trompeur dans la mesure où il tend à naturaliser un fait d'essence sociale, à isoler comme pathologie 
un phénomène qui concerne la redéfinition du rapport aux autres et à la règle sociale. Les spécialistes définissent l'addiction ${ }^{1}$ comme « une condition selon laquelle un comportement susceptible de donner du plaisir et de soulager des affects pénibles est utilisé d'une manière qui donne lieu à deux symptômes-clés : échec répété de contrôler ce comportement ; poursuite de ce comportement malgré ses conséquences négatives ». L'addiction signifie donc l'impossibilité pour la personne concernée de se contrôler. Or c'est l'ambivalence voire le paradoxe qui caractérise les pratiques de jeux vidéo, du moins un certain nombre d'entre elles. Si elles témoignent d'une recherche et d'un apprentissage de contrôle de soi, du monde virtuel, elles font simultanément écho à un rapport d'aliénation au monde et au refus des contraintes de la vie sociale moderne dans leur recherche de maîtrise au monde. Ces pratiques se signalent alors par une perte de contrôle. Le sentiment de toute puissance se conjugue avec celui d'impuissance, l'évasion se confond avec l'addiction, ou la répétition automatique, les moyens empruntés s'avérant être de même nature que le cercle de contraintes qu'il s'agit de briser.

«Il y a l'idée de se dominer soi-même pour dominer le jeu. » nous dit un joueur sur téléphone portable. Aux commandes de la machine, il faut domestiquer son corps et ses réflexes pour triompher des obstacles de la partie. À terme, cela procure un sentiment de puissance. Le jeu vidéo peut servir d'exutoire à toutes les frustrations de la vie en société. «Je vais prendre l'exemple du métro. On est dans le métro. Les gens reviennent $d u$ boulot, ils sont tous énervés, ils puent, ils toussent, c'est la folie. Vous, vous êtes à 6 sur les banquettes. Et tout le monde est là à se crier dessus, se bousculer. C'est la jungle. Les gens sont ternes, tout blancs... Vous, vous prenez votre téléphone. Vous voyez sur le jeu des petites formes, des ronds, des bruits : « bip, bip, bip ! » $C$ 'est ça s'évader » dit un joueur. L'attention que nécessite le jeu permet de détourner sa sensibilité de la gêne liée à la présence de l'autre. Avec le jeu sur portable, le joueur possède un pouvoir : celui d'apparaître ou de disparaître du monde selon sa volonté.

La pratique de ces jeux renvoie l'individu sommé de devenir acteur de sa propre $v^{2} e^{2}$ au principe d'un contrôle de soi en même temps qu'à de nouveaux modes d'être ensemble. L'explosion des addictions ne fait-elle pas écho à cette nouvelle norme sociale de la responsabilité et de l'initiative dont on sait le lien qu'elle entretient avec la dépression, cette maladie de la modernité ${ }^{3}$ ? L'effacement du corps, la dégradation des sens en stimulations sensorielles signalent une perte de l'engagement de soi envers la société. Sans acte de présence, le sentiment d'obligation morale se dissipe : quels devoirs a-t-on envers une société susceptible de disparaître et de réapparaître au gré des connexions informatiques?

Livrés seuls à nous-mêmes, c'est la figure de l'anomie signalée en son temps par Durkheim qui ressurgit alors, lorsque les appétits ne sont plus contenus par une opinion désorientée et ne savent plus où sont les bornes devant lesquelles ils doivent s'arrêter. "Tout se passe comme si, écrit Durkheim, on était stérilement agité sur place (...) Poursuivre une fin inaccessible par hypothèse, c'est donc se condamner à un perpétuel état de mécontentement ${ }^{4}$. Avec ce « mal de l'infini » qui caractérise l'anomie, les 
joueurs de jeux vidéo sont menacés de perdre le plaisir qui justifiait au départ leur pratique. Le milieu du jeu professionnel en témoigne : le jeu devient un travail pour lequel seul l'instant de la victoire vaille que l'on consacre des heures d'entraînement ${ }^{5}$.

\section{Recherche de puissance et désir de contrôle}

Pourtant le jeu incite en même temps à une discipline de soi. Celle-ci est d'autant plus rigoureuse que la capacité d'attraction du jeu est importante. Deux logiques s'affrontent ici : la maîtrise du jeu et le contrôle de soi. Les joueurs que nous avons rencontrés accordent une grande place à la nécessité de se réguler eux-mêmes, ils insistent sur leur nécessaire capacité de "selfcontrol» autant que sur leurs performances ludiques. La figure du «no-life», le joueur tellement absorbé dans son univers ludique qu'il n'est plus vraiment vivant, d'après les termes utilisés par les joueurs eux-mêmes, agit comme un repoussoir, une figure dont chacun cherche à se démarquer. L'efficacité des procédés d'addiction du jeu est alors mise en cause.

La contradiction n'est toutefois qu'apparente. Si l'on s'intéresse à la formulation de ces plaidoyers de joueurs qui défendent la nécessité d'une vie sociale en dépit du jeu, on constate qu'ils parlent de sociabilité en termes de performance: «Je n'ai jamais souhaité gérer une Guilde, parce que j'ai vu très rapidement que c'était beaucoup d'implications. Parce que ça ne s'arrête pas simplement au fait de gérer une Guilde en jeu, il y a aussi tout l'aspect Forum qui prend énormément de temps, et clairement, je n'avais pas de temps à consacrer à ça, et je n'avais pas non plus l'envie de gérer une communauté directement ». La sociabilité est un enjeu, au même titre que ceux de la partie. Le temps apparaît comme une valeur économique, que l'on investit. Temporalité de l'instant, de l'immédiat, dans lequel la gestion de l'autre se substitue imperceptiblement à la disponibilité nécessaire à l'élaboration d'un lien social. «Dans le mondecomme-jeu, le temps se divise en une succession de parties. Chacune se compose de ses propres conventions; chacune est une "province de signification » distincte - un petit univers en soi, indépendant et fermé sur lui-même $»^{6}$. Dans une temporalité divisée en instants, la perception de l'autre est morcelée. Elle ne s'atteint d'abord qu'à travers les enjeux de la partie.

Ces jeux reposent sur des caractéristiques correspondant à ce que Csikszentmihalyi appelle «état optimal de l'expérience» ou flow? ${ }^{7}$, cette expérience d'une activité qui constitue sa propre gratification et qui n'exige pas de finalité. Dans ce cas l'attention est plus centrée sur l'action elle-même que sur ses effets. L'intérêt, voire la passion que suscitent les jeux sur Internet sont, nous le voyons, directement liés à leurs caractéristiques techniques combinées à des phénomènes sociaux typiques de notre modernité: la recherche de performance, la prépondérance du temps présent, immédiat, voire de l'urgence, la recherche de sensations ${ }^{8}$.

Si la liberté est une constante fondamentale des jeux traditionnels - elle est ce qui définit l'activité ludique dans son désintéressement, son aptitude pédagogique à enseigner par le plaisir certaines conventions sociales majeures - nous pouvons d'ores et déjà avancer que le jeu 
vidéo met à mal cette définition en imposant un rapport essentiellement centré sur la maîtrise et l'efficacité. Celles-ci reposent sur les techniques de résolution de problèmes", qui peuvent s'apparenter à du bricolage, comme l'indique Benoît : «On a besoin de bricoler, on a besoin d'outils, bon, ben, voilà, on va chercher l'outil! (...) C'est un peu ça, oui. À chaque fois qu'on a quelque chose qui nous pose problème, on va trouver la solution et résoudre ce problème. ", il s'agit de trouver à chaque fois les bonnes procédures. Les enjeux successifs imposés par la structure labyrinthique de ces programmes imposent au joueur une avancée linéaire encombrée par de nombreux obstacles sous forme d'énigmes ou d'autres épreuves fondées sur la maîtrise des commandes.

Ce sentiment de puissance donné par le jeu vidéo et la pratique d'Internet vient tout particulièrement de l'extension de la capacité d'action à laquelle il donne accès et, dans le cas de certains jeux, des sensations procurées, qui simulent l'action. "C'est bien ce qu'ont compris certains concepteurs de jeu, comme celui qui dit avoir découvert le caractère addictif des manifestations sonores accompagnant les actions des joueurs: quand je clique ça fait pock, ça supprime, moi je faisais pock pock pock pock, pock pock pock pock... le joueur, quand je l'avais fait tester, avait une sensation justement de puissance très forte. Il avait une sensation de détruire et en fait c'est le jeu qui a le mieux marché... finalement c'était juste sur un tout petit bruit.... ça fait du bruit et on est le maître du monde ».

Il y a pathologie dans la pratique des jeux vidéo lorsque le joueur s'engage «dans le cauchemar d'un contrôle omnipotent $»^{10}$. La pathologie vient avec le désir de contrôle absolu.

Finalement, l'attrait du jeu vidéo ne vient-il pas particulièrement de ce qu'il combine illusion de contrôle, voire de puissance, et de maitrise du sens $^{11}$ ? Car ces jeux se caractérisent par les règles sur lesquelles ils reposent, règles que l'on peut difficilement ignorer ou outrepasser puisqu'elles sont inscrites dans le système informatique. C'est finalement le monde de la simulation qui est le propre de ces jeux : «la philosophie du jeu Donjons et Dragons, avec ses livres de règles, semble plus attrayante et stimulante que l'histoire de la vraie vie et que les jeux de fiction dont les règles sont moins précises $»^{12}$. La seule obligation dans ces jeux, écrit Turckle, est la logique. Voici enfin un monde cohérent, que l'on peut expliquer, qui répond à des règles connaissables. Les jeux vidéo sont spécifiques parce qu'ils reposent sur des programmes informatiques dont une caractéristique essentielle est d'être logique et cohérent. "Contrairement à l'univers des flippers, des sports ou de la littérature, les programmes qui se trouvent à l'intérieur des machines de jeu vidéo en font des jeux 'dirigés par des règles'. Le joueur sait que le programme détient la clé des secrets ». Certains types de jeux sont appréciés parce qu'il y a un début et une fin, que le concepteur connait. «Pas de risque alors de basculer $»$ dit Benoît. Ce monde ludique est clair et simple, sans incertitude majeure. L'attraction pour ces jeux vient des règles mêmes qui sont inscrites dans les programmes, donnant à la fois un sentiment de contrôle de soi et du monde. Ainsi le fait nouveau est moins l'absence de règle que la toute puissance prêtée à la règle une fois celle-ci matérialisée dans des 
procédures et des algorithmes.

\section{Recherche de sensations et recherche de sens}

Ehrenberg parle du cyberespace comme «machine à relation et machine à sensations qui offre la possibilité de créer sa propre réalité grâce aux techniques du virtuel, ce mariage de déstabilisation des perceptions, que procure la drogue depuis longtemps, et du spectacle de la réalité $\rangle^{13}$. On voit bien la proximité qui peut s'établir d'entrée de jeu entre drogue et usage excessif d'Internet et des jeux vidéo. Mais c'est aussi ce qui, paradoxalement, les distingue. La dépendance aux jeux vidéo diffère de celle aux jeux d'argent ou aux drogues car «le joueur vidéo serait plus 'interactivo-dépendant', c'est-à-dire dépendant de la fantastique interactivité qu'il établit avec sa machine $»^{14}$. L'attrait de ces jeux repose en grande partie sur leur capacité à procurer des émotions, des sensations ${ }^{15}$, un sentiment d'action, voire de puissance, sur l'absence de limite, comme c'est le cas pour les univers persistants. Si ces dimensions nous renvoient à l'addiction et à son approche psychologique, voire psychiatrique, elles ouvrent aussi d'autres perspectives, socioanthropologiques, cette fois.

La croyance est l'une des principales caractéristiques de la pratique ludique d'après Huizingua. Le jeu est «une activité absorbante » dont les enjeux apparaissent d'autant plus sérieux qu'ils nécessitent de se battre pour eux avec vigueur. Bien qu'elle semble recouvrir essentiellement des pratiques de jeux agonales, cette définition met en lumière l'importance de l'adhésion du joueur dans son activité. Le jeu vidéo est une «fabuleuse machine de propagande $»^{16}$. Il s'avère en effet que l'efficacité de l'immersion dans le jeu peut favoriser en retour une adhésion à des doctrines institutionnelles aux implications sérieuses, pour ne pas dire « réelles »: «Monde quotidien et jeu s'assistent mutuellement. Par les émotions, le jeu renforce la croyance aux principes d'organisation non seulement $d u$ jeu, mais aussi en ceux du monde quotidien (...) Plus l'effet de réel d'un jeu est fort, plus l'intérêt aux principes organisateurs que le jeu et le monde quotidien ont en commun est fort $»^{17}$. Dès lors, il apparaît que la croyance est un marqueur sociologique dans les pratiques de jeu, au sens où il transmet un « effet de réel » et supporte un phénomène mimétique. À travers la croyance, c'est l'émotion du joueur en général qu'il convient d'interroger. Non pas tant ce qui la suscite que ce qu'elle fait naître comme valeurs nouvelles. «Plus la vie quotidienne exige des individus de contenir leurs sentiments et l'expression corporelle, et plus les jeux prennent une part importante dans la quête d'émotions fortes ${ }^{18}$. D'où l'importance de la notion de « défoulement », « se vider la tête après le travail », mais dans une démarche somme toute perverse, puisque cette détente, cette relaxation renvoie aux mêmes logiques auxquelles le joueur tente de s'émanciper.

Certains auteurs montrent l'importance de la recherche de sensations dans l'addiction, en particulier avec les jeux vidéo ${ }^{19}$. Les joueurs qui recherchent l'excitation « sont probablement les plus menacés par le risque de devenir accros aux jeux vidéo, car ceux-ci sont comparables pour eux à n'importe quel autre toxique ». « Selon ce qu'il privilégie, écrit encore Tisseron, le joueur de jeux vidéo s'engage soit du côté du bain d'excitations, soit du côté de sa maîtrise et de sa mise en sens ». 
Cette recherche de "mise en sens" est très récurrente (Craipeau et alii, 2002, 2005). C'est ici que l'on peut d'ailleurs considérer la dimension sociologique, voire anthropologique majeure de ces jeux : ils correspondraient particulièrement aux caractéristiques de nos sociétés industrialisées, dans lesquelles le corps n'est plus tant le médiateur avec le monde réel mais devient le réel immédiat ${ }^{20}$. Les stimulations sensorielles aménagent une place au corps en même temps qu'elles en contrôlent les débordements dans une société marquée par l'extrême sédentarité. À travers ces nouvelles pratiques de jeu c'est l'immobilité du corps attachée aux activités tertiaires et ses conséquences (le sentiment de déréalisation de soi et du monde) qu'il s' agit sinon de compenser, du moins de conjurer. Et les effets n'en sont pas moins délétères. Ces jeux sont alors un moyen de combler l'ennui, le vide en soi, ou bien, paradoxalement, permettent d'évacuer une tension, un trop plein et d'une certaine façon de créer un vide considéré comme reposant. La recherche de sensations se trouve très souvent associée à une recherche de puissance, ou de maîtrise de soi, de l'environnement, voire des autres (Craipeau et Dubey 2006, Roustan 2005).

L'absence du corps dans la relation qui s'établit entre joueurs (sauf dans le cas où ils peuvent dialoguer oralement) n'est pas sans effet sur la nature de la relation elle-même. «En absorbant une autre personne par le regard, on se révèle soi-même, écrit Simmel ${ }^{21}$, par la même action le sujet, tout en cherchant à reconnaître l'objet, se livre à lui. On ne peut pas prendre par l'œil sans donner en même temps, car l'œil dévoile à celui qui regarde l'âme qui cherchait à le dévoiler ». Jouer derrière son écran, n'est-ce pas aussi con- trôler, ou chercher à contrôler la relation à l'autre, en évitant de s'exposer trop directement ?

Les jeux vidéo seraient des laboratoires sociaux dans lesquels les joueurs testeraient leur capacité de contrôler leur monde virtuel, à défaut de contrôler leur vie, répondant ainsi à l'injonction sociale d'autonomie et de contrôle dans laquelle ils sont éduqués. Car l'a-normalité maintenant stigmatise ceux qui ne peuvent se contrôler. Des chercheurs américains indiquent que « les ÉtatsUnis sont addicts à l'addiction, que la maladie $d u$ siècle est la perte de contrôle sur soi $\rrbracket^{22}$. Et c'est cette recherche de contrôle et l'illusion d'y parvenir qui est source de dépendance, éventuellement d'addiction. Phénomène auquel participe actuellement l'offre de l'industrie du jeu vidéo. Mais cela est-il univoque?

\section{Conclusion}

Les véritables émancipations à ces logiques de production sont donc à chercher sur un tout autre plan : celui du social, tel qu'en parle Durkheim. Tandis que le jeu vidéo propose un monde à l'échelle de l'individu, le social le transcende, pour le réguler, lui imposer une réalité nourrie de l'expérience de chacun, en une entité indépendante. Paradoxalement, c'est dans ce passage à la sphère du social que l'addiction au jeu se ressent le plus fortement. Il faut cette résistance du corps social pour éprouver les limites de cette maîtrise de soi. Les limites du jeu vidéo sont souvent celles du corps humain : une fatigue se fait sentir à mesure que l'on s'investit dans les mécanismes d'addiction : " J'ai l'impression que ce sont des images qui se répètent dans la tête. Dans les rêves en général, ça me rend extrêmement nau- 
séeuse. Je me réveille, je suis toute aussi crevée que quand je me suis couchée. C'est vraiment terrifiant. Donc, quand on arrive à ce point, on dit: «Stop! Là, je vais me balader en forêt. $C$ 'est bon. Je fais une semaine je ne joue plus. » Il faut savoir s'arrêter. ». Quelles que soient leurs pratiques, les joueurs font état d'une fatigue de jouer, une frustration d'autant plus lourde que les heures de jeux auront été intenses.

«Ce qui résiste et par là même s'affirme, c'est bien la dimension affective et sensible de notre rapport au monde. Derrière ce constat d'apparence anodine se joue pourtant quelque chose d'essentiel, une autre version du monde où la capacité d'être affecté, touché par autrui, acquiert la légitimité d'un mode de connaissance à part entière $»^{23}$.

Les jeux vidéo dessinent une nouvelle façon d'être au monde, plus urgente, plus instable, mais aussi plus sensible à l'intense fragilité de notre existence. Les nouvelles technologies, en nous plongeant dans un état de fascination, de quasi-dépendance à la technique, dans son immédiateté, son confort, nous confronteraient à la vacuité de notre expérience sensible. Reste entière la question de la création des formes sociales capables de structurer ces expériences. L'espace des jeux vidéo se présente ainsi comme ce lieu transitoire où s'expérimentent, depuis l'expérience de l'anomie, de nouvelles règles et de nouvelle modalités de l'être ensemble.
$\mathrm{N}$

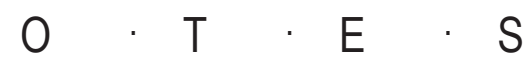

1. Ades J., in rapport Inserm à paraître 2008, « Le jeu excessif ».

2. Ehrenberg A., L'individu incertain, Pluriel Calmann-Lévy, 1995.

3. Ehrenberg A., La fatigue d'être soi, poche Odile Jacob, 1998 .

4. Durkheim E., Le Suicide, PUF, 1930, p. 281.

5. «Les joueuses que j'entraîne n'ont quasiment plus aucun plaisir à pratiquer le jeu. Le plaisir, comme toutes sportives de haut niveau, elles le tirent de la victoire, de l'achèvement, de la progression. Elles ne le tirent pas de la pratique du jeu. »: Entretien avec Jean-Baptiste, 30 ans, coach d'une équipe professionnelle $\mathrm{du}$ jeu en réseau Counterstrike, 17/07/2006.

6. Bauman Z., La Vie en miettes, Le Rouergue/ Chambon, 2003, p. 56.

7. Csikszentmihalyi, Flow, the Psychology of Optimal Experience, New York Harper and Row, 1990.

8. Balandier G., Le Grand Système, Fayard, 2001.

9. Résoudre un problème, c'est à la fois évoquer des actions et des procédures associées à une situation et construire une interprétation de la situation qui soit cohérente avec la mise en œuvre de ces savoir-faire (mathématiques, psychologie cognitive).

10. Tisseron S., «Les enjeux d'une passion», Dépendances, $\mathrm{n}^{\circ} 28$, avril 2006, SFA/ISPA.

11. On retrouve ici les «techniques du bonheur» préconisées, dans une logique behavioriste, par Csikszentmihalyi, qui propose les moyens de trouver du sens dans les activités qui nous mobilisent.

12. Sherry Turkle, Les enfants de l'ordinateur, Denoël, 1986.

13. Ehrenberg A. L'individu incertain, CalmannLévy, Paris, 1995, p. 275. 
14. Tisseron S., op. cit., 2006

15. Craipeau S., Dubey G., «The ways mobile telephone games are used: marking out sociability spaces and "taking back control" of time ", Institut National des Telecommunications, Groupe des Écoles de Telecommunications, Helsinki, June 2006.

16. Stéphane Natkin Directeur de l'ENJMIN : École Nationale des Jeux et des Médias Interactifs Numériques, située à Angoulême.

17. Gebauer G., Wulf C., Jeux, rituels, gestes : les fondements mimétiques de l'action sociale, Anthropos, Economica, 2004, p. 176.

18. Gebauer et Wulf, op. cit. , p. 181.

19. Laving M., Marvin K., Mclarney A., Nola V., Scott L., «Sensation seeking and collegiate vulnerability to internet dependence», Cyber Psychology and Behavior, vol 2, n5, 1999.

20. Balandier G., Le grand système, Fayard 2001.

21. Simmel G., « Essai sur la sociologie des sens », in Sociologie et épistémologie, PUF, 1980.

22. Baumeister R, Vohs K.D., Handbook of Self-Regulation, research, theory and applications, The Guilford Press New York, London, 2004.

23. Dubey G., Le lien social à l'ère du virtuel, PUF, 2001, p. 213. 